\title{
Divertículo colónico gigante: Dos nuevos casos en la literatura
}

\section{Giant Colonic Diverticulum: Two New Cases in Literature \\ Divertículo gigante de colom: Dois novos casos na literatura}

\author{
María Alejandra Baquero-Serrano, MD. * \\ Luis Andrés López-Martínez, MD. ** \\ Federico Guillermo Lubinus-Badillo, MD., Esp. *** \\ Eduardo Alberto Higuera-Escalante, MD., Esp. *** \\ Carlos Alfonso Rey-Grass, MD., Esp. ***
}

\begin{abstract}
Resumen
Introducción: El divertículo colónico gigante es una presentación rara de la enfermedad diverticular del colon descrita por primera vez en la literatura francesa en 1946; se define cuando su diámetro es mayor a $4 \mathrm{~cm}$. Objetivo: Adicionar a la literatura dos casos diagnosticados en la Fundación Oftalmológica de Santander, Colombia debido al bajo número de casos reportados en la actualidad; además, mencionar los principales datos encontrados en la literatura en cuanto a la epidemiología, la presentación clínica, los diagnósticos diferenciales, las herramientas diagnósticas más importantes a tener en cuenta y la diferencia de los esquemas de tratamiento. Presentación de los casos: Se presentan dos casos de pacientes con clínica de dolor abdominal para los cuales se solicitaron estudios imagenológicos que permitieron realizar un adecuado diagnóstico de divertículo colónico gigante; el primero fue manejado de manera conservadora con antibiótico-terapia y el segundo de forma quirúrgica con resección de colon transverso con anastomosis primaria. Conclusiones: El
\end{abstract}

divertículo colónico gigante es una entidad poco frecuente y por tanto escasamente descrita en la literatura asociada en su mayoría con la diverticulosis. Se presenta con una clínica muy diversa, sugestiva de múltiples patologías abdominales que deben ser descartadas para poder realizar un adecuado diagnóstico por medio de técnicas de imagen como la radiografía, el colon por enema y la tomografía axial computarizada que representan las principales herramientas diagnósticas de esta entidad. El tratamiento de elección es la intervención quirúrgica, sin embargo, en contextos determinados como pacientes ancianos, asintomáticos y con alto riesgo quirúrgico puede manejarse de manera conservadora. Su importancia a pesar de la baja prevalencia radica en la necesidad del diagnóstico oportuno para evitar una alta tasa de complicaciones. [Baquero-Serrano MA, López-Martínez LA, Lubinus-Badillo FG, Higuera-Escalante EA, Rey-Grass CA. Divertículo colónico gigante: Dos nuevos casos en la literatura MedUNAB 2017; 20(2): 235-243].

Palabras Clave: Divertículo; Divertículo del Colon; Dolor Abdominal; Radiografía Abdominal; Tomografía.

* Médica, Residente de Radiología e Imágenes Diagnósticas, Facultad de Ciencias de la Salud, Universidad Autónoma de Bucaramanga (UNAB), Bucaramanga, Santander, Colombia.

** Médico general, Facultad de Ciencias de la Salud, Universidad Autónoma de Bucaramanga (UNAB), Bucaramanga, Santander, Colombia.

${ }^{* * *}$ Médico, especialista en Radiología e Imágenes Diagnósticas, Servicio de Radiología, Fundación Oftalmológica de Santander - Clínica Carlos Ardila Lulle (FOSCAL), Bucaramanga, Santander, Colombia

Correspondencia: MaríaAlejandra Baquero Serrano. Teléfono: +573166916569 E-mail: mbaquero@unab.edu.co 


\section{Abstract}

Introduction: The giant colonic diverticulum is a rare presentation of the diverticular disease of the colon described for the first time in French literature in 1946 and it is defined when its diameter is greater than $4 \mathrm{~cm}$. Objective: To add to the literature two more cases diagnosed in the Ophthalmological Foundation of Santander, Colombia due to the low number of cases reported today; in addition, to mention the main data found in the literature in terms of epidemiology, clinical presentation, differential diagnoses, the most important diagnostic tools to consider and the difference of treatment schemes for it. Presentation of the cases: Two cases of patients with abdominal pain clinic for whom imaging studies were requested that allowed an adequate diagnosis of giant colonic diverticulum are presented. The first one was managed conservatively with antibiotic therapy and the second one was surgically treated with a transverse colon resection with primary anastomosis. Conclusions: The giant colonic diverticulum is a rare entity and therefore poorly described in the literature associated mostly with diverticulosis. It is presented with a very diverse clinic, suggestive of multiple abdominal pathologies that must be discarded in order to perform an adequate diagnosis by means of imaging techniques such as x-rays, colon by enema and computerized axial tomography scan which represent the main diagnostic tools for this entity. The treatment of choice is surgery; however, in determined contexts such as with elderly patients who are asymptomatic and with a high surgical risk can be managed conservatively. Its importance despite its low prevalence lies in the need for timely diagnosis to avoid a high rate of complications. [Baquero-Serrano MA, López-Martínez LA, Lubinus-Badillo FG, Higuera-Escalante EA, Rey-Grass CA. Giant Colonic Diverticulum: Two New Cases in Literature. MedUNAB 2017; 20(2): 235-243].

Keywords: Diverticulum; Diverticulum, Colon; Abdominal Pain; Radiography, Abdominal; Tomography.

\section{Introducción}

Los divertículos del colon son una entidad crónica caracterizada por la protrusión sacular de la mucosa a través de áreas débiles de la pared muscular (1). Es una enfermedad común no influenciada por el género, localizada en el 95\% de los casos en el colon sigmoides. Según la Organización Mundial de Gastroenterología la prevalencia de enfermedad diverticular en el mundo varía entre 5-45\% y aumenta con la edad afectando en más del $60 \%$ a la población mayor de 70 años $(2,3)$. La patogénesis de esta entidad resulta de la interacción entre la baja ingesta de fibra, la debilidad de la pared colónica y la disminución de la motilidad intestinal (4).

Una presentación rara de esta entidad es el divertículo colónico gigante descrito por primera vez en Francia por Bouvin y Bonte en 1946 (5,6). Una revisión sistemática publicada en el año anterior (1945) incluyó un total de 166 casos identificados en 138 estudios por lo que desde 1946 se

\section{Resumo}

Introdução: O divertículo colônico gigante é uma apresentação rara da doença diverticular do cólon descrita pela primeira vez na literatura francesa em 1946; é definido quando seu diâmetro é maior que $4 \mathrm{~cm}$. Objetivo: Adicionar à literatura dois casos diagnosticados na Fundação Oftalmológica de Santander, Colômbia devido ao baixo número de casos relatados no presente; Além disso, mencionar os principais dados encontrados na literatura em termos de epidemiologia, apresentação clínica, diagnósticos diferenciais, as ferramentas de diagnóstico mais importantes a serem consideradas e a diferença de esquemas de tratamento. Apresentação dos casos: Foram apresentados dois casos de pacientes com clínica de dor abdominal para os quais foram solicitados estudos de imagem que permitiram um diagnóstico adequado de divertículo colônico gigante; O primeiro foi administrado de forma conservadora com terapia antibiótica e o segundo foi tratado cirurgicamente com ressecção do colo cruzado com anastomose primária. Conclusões: Divertículo colônico gigante é uma entidade rara e, portanto, pouco descrita na literatura associada principalmente à diverticulose. É apresentado um quadro clínico muito diverso, sugestivo de múltiplas patologias abdominais que devem ser descartadas para poder fazer um diagnóstico adequado por meio de técnicas da imagem como a radiografia, o cólon por enema e tomografia axial computorizada que representam as principais ferramentas Diagnóstico desta entidade. O tratamento a escolher é a cirurgia, no entanto, em determinados casos como é a dos pacientes idosos, assintomáticos e com alto risco cirúrgico podem ser tratados de forma tradicional. Sua importância, apesar da baixa prevalência, reside na necessidade de um diagnóstico oportuno para evitar uma alta taxa de complicações. [Baquero-Serrano MA, LópezMartínez LA, Lubinus-Badillo FG, Higuera-Escalante EA, ReyGrass CA. Divertículo gigante de colom: Dois novos casos na literatura. MedUNAB 2017; 20(2): 235-243].

Palavras chave: Divertículo; Divertículo de Colo; Dor Abdominal; RadiografiaAbdominal; Tomografia.

han reportado menos de 200 casos en la literatura (7). Se define el divertículo colónico gigante (DCG) cuando el diámetro es mayor de $4 \mathrm{~cm}$, teniendo en cuenta que en la literatura se encuentran descritos hasta de $40 \mathrm{~cm}(5,6)$. En la mayoría de los casos el DCG es único, pero puede ser múltiple, afectando principalmente a personas mayores de 60 años. No muestra predilección por ningún género y aproximadamente el $90 \%$ de los casos se presenta en el colon sigmoides, casi siempre localizado en el borde antimesenterico (95\%) $(4,8)$. En el $85 \%$ de los casos se asocian con enfermedad diverticular, aunque pueden presentarse de manera aislada en un $15 \%(7,9)$.

Su etiología es desconocida, pero según la mayoría de las revisiones la teoría más aceptada se basa en una válvula unidireccional en el cuello del divertículo que permite el paso de gas dentro de este, lo que provoca una distensión progresiva de su pared. La segunda teoría más aceptada consiste en microorganismos intestinales productores de gas contenidos dentro del divertículo lo que provoca un aumento de su tamaño $(4,7)$. El objetivo de este trabajo fue reportar 
dos casos diagnosticados en la Clínica FOSCAL (Santander, Colombia), cuyo interés radica en la escasa frecuencia de presentación y el bajo número de casos reportados en la literatura mundial. Además, mencionar los principales datos encontrados en la literatura en cuanto a la epidemiología, la presentación clínica, los diagnósticos diferenciales, las herramientas diagnósticas más importantes a tener en cuenta y la diferencia de los esquemas de tratamiento.

\section{Presentación de los casos}

En este artículo se contó con la aplicación del consentimiento informado por medio del cual los dos pacientes presentados dieron su autorización para la utilización y publicación de su historia clínica y herramientas diagnósticas usadas como las imágenes presentadas.

\section{Caso número 1}

Paciente masculino de 55 años con antecedente de mieloma múltiple con plasmocitoma en húmero izquierdo y neuropatía periférica severa, candidato a trasplante autólogo de progenitores hematopoyéticos quien ingresó para iniciar protocolo de acondicionamiento pre-trasplante. $\mathrm{Al}$ ingreso se encontró paciente asintomático. Al examen físico abdomen blando, no masas, sin signos de irritación peritoneal.

Posteriormente el paciente inició con astenia, adinamia, deposiciones diarreicas abundantes, picos febriles asociados a taquicardia y dolor abdominal leve a la palpación en fosa iliaca derecha y flanco derecho con aumento de reactantes de fase aguda. Debido a su patología de base se inició manejo antibiótico de amplio espectro con meropenem y vancomicina y se solicitaron estudios complementarios.

Se realizó Tomografía Axial Computarizada (TAC) de abdomen contrastado en donde se visualizó una dilatación sacular en la región del ángulo hepático del colon ascendente la cual midió $7.0 \mathrm{~cm}$ de diámetro antero posterior, $5.5 \mathrm{~cm}$ longitud y $6.2 \mathrm{~cm}$ transverso. Esta lesión se encontraba ocupada casi en su totalidad por materia fecal (Figura 1) y presentaba un cuello de $31.0 \mathrm{~mm}$ de diámetro (Figura 2 y 3), hallazgo compatible con divertículo gigante del colon, además, presentaba ligero engrosamiento de la pared y de la grasa circundante, sin embargo, no se observó gas libre en el abdomen.

En consecuencia, se decidió suspender vía oral, continuar con antibiótico-terapia y se inició nutrición parenteral con posterior mejoría del dolor abdominal. Posteriormente fue valorado por el servicio de cirugía general quienes encontraron paciente con resolución del dolor abdominal, tolerando vía oral por lo que se continuó manejo

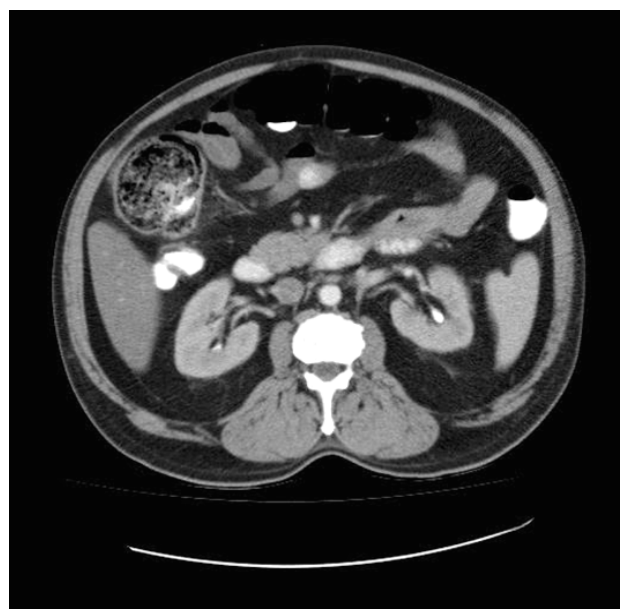

Figura 1. Tomografía Axial Computarizada de abdomen contrastado. Corte axial que muestra divertículo colónico gigante ocupado por materia fecal (Caso número uno)

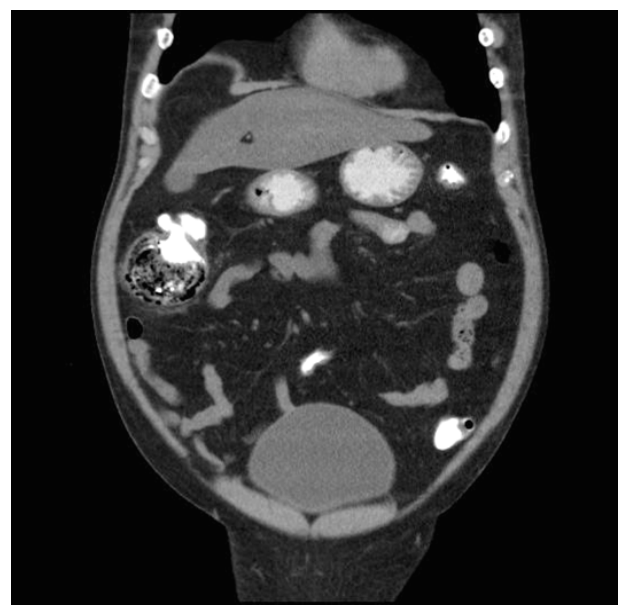

Figura 2. Tomografía Axial Computarizada de abdomen contrastado. Corte coronal. Cuello del divertículo colónico gigante de $31 \mathrm{~mm}$ de diámetro (Caso número uno).

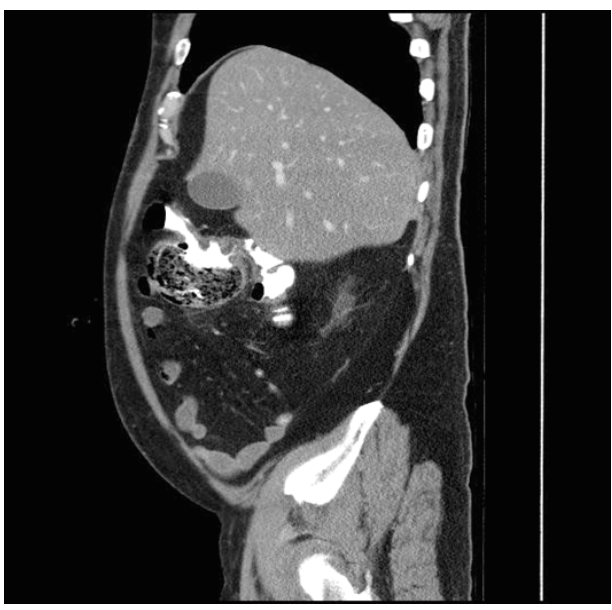

Figura 3. Tomografía Axial Computarizada de abdomen contrastado. Corte sagital. Cuello del divertículo colónico gigante de $31 \mathrm{~mm}$ de diámetro (Caso número uno). 
conservador y finalmente por estabilidad hemodinámica, resolución de su cuadro abdominal y por presentar recuperación hematopoyética, se dio de alta hospitalaria sin complicaciones.

\section{Caso número 2}

Paciente femenino de 65 años de edad con antecedentes de linfoma del manto en manejo con quimioterapia, anemia severa, diabetes mellitus tipo 2 e hipotiroidismo primario. Ingresó por cuadro clínico de 2 días de evolución consistente en dolor abdominal difuso asociado a náuseas, distensión abdominal, picos febriles y emesis en múltiples episodios con deterioro del estado general. Al examen físico, se encontró la paciente en regulares condiciones generales, álgica, estable hemodinámicamente, abdomen distendido, blando con dolor sordo de intensidad moderada a la palpación de epigastrio e hipocondrio izquierdo y adenopatías inguinales bilaterales induradas no dolorosas.

La paciente es ingresada con indicación de observación, manejo con hidratación endovenosa, analgesia y antiemético, se solicitaron paraclínicos en los que se evidenció bicitopenia y trastornos hidroelectrolíticos asociados (hipomagnesemia, hipocloremia e hipercalcemia) con elevación de fosfatasa alcalina, amilasa y bilirrubina directa. Se realizó ecografía abdominal que mostró a nivel del hipocondrio izquierdo imagen de masa, con abundante sombra acústica posterior y que no permitía su adecuada valoración.

Fue valorada por servicio de cirugía general por exacerbación de la sintomatología dada por emesis persistente, distensión abdominal y deposiciones diarreicas de características inflamatorias quienes encontraron al examen físico signos de irritación peritoneal y masa de aproximadamente $5 \mathrm{~cm}$ de diámetro localizada entre la región de epigastrio e hipocondrio izquierdo, de consistencia dura, dolorosa a la palpación, de bordes bien definidos por lo que iniciaron manejo antibiótico endovenoso con piperacilina tazobactam y metronidazol y solicitaron TAC de abdomen contrastado.

La TAC reportó múltiples imágenes diverticulares en el colon (Figura 4), se observó una imagen, un divertículo gigante del colon localizado hacia la unión del colon transverso con el ángulo esplénico el cual medía $6.0 \mathrm{~cm}$ de diámetro y se encontraba opacificado con restos de materia fecal y gas sin mayores signos de sobreinfección (Figura 5).

Se sugirió practicar colon por enema, el cual permitió visualizar, según la placa simple, una imagen sugestiva de materia fecal impactada, hacia el cuadrante superior izquierdo del abdomen (Figura 6). Se administró medio de contraste baritado, observándose abundante cantidad de imágenes diverticulares, las cuales dependían de todos los bordes del mesenterio y se distribuían desde el recto sigmoides hasta el ciego (Figura 7).

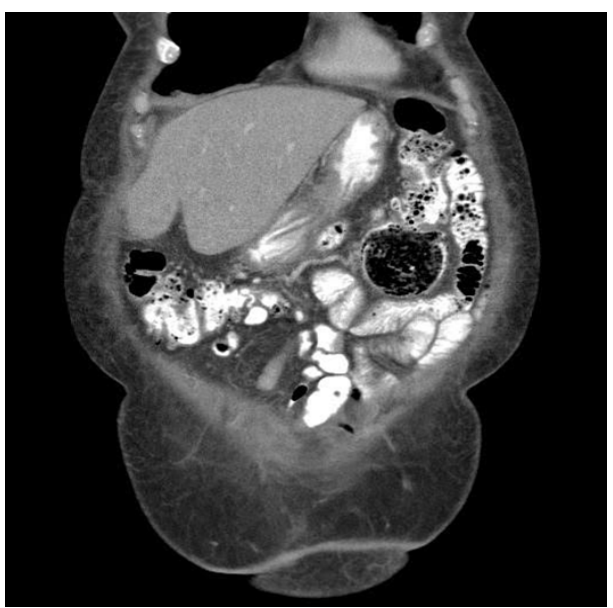

Figura 4. Tomografía Axial Computarizada de abdomen contrastado. Corte coronal. Múltiples dilataciones saculares en todo el colon (Caso número dos).

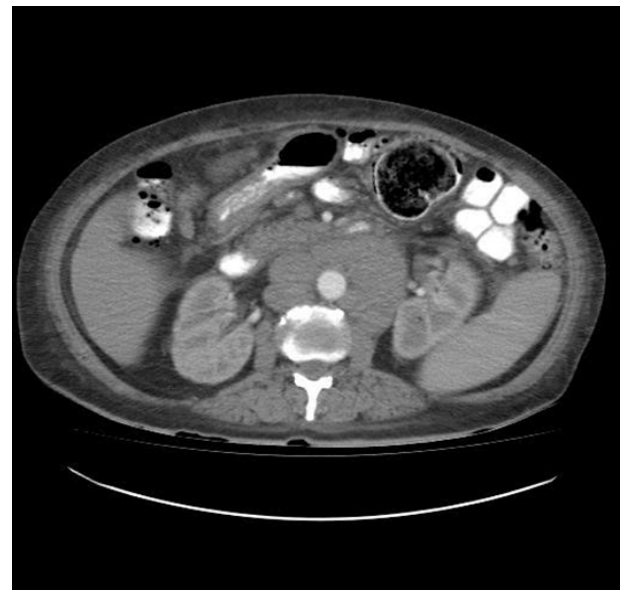

Figura 5. Tomografía Axial Computarizada de abdomen contrastado. Corte axial. Divertículo colónico gigante con restos de material fecal y gas en su interior (Caso número dos).

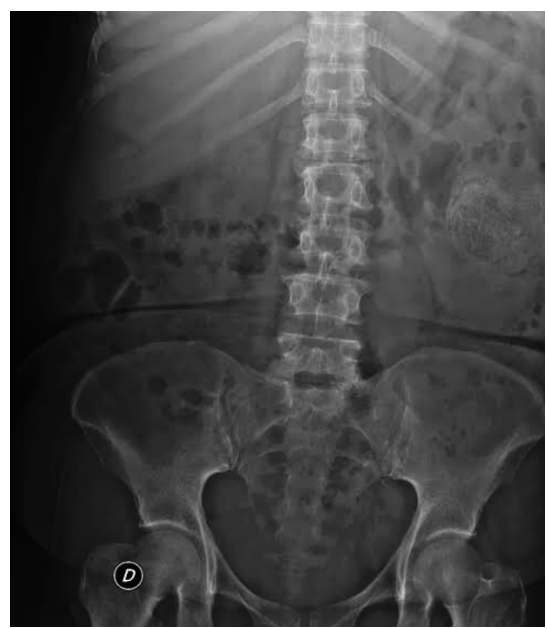

Figura 6. Radiografía simple de abdomen. Imagen sugestiva de materia fecal impactada en el cuadrante superior izquierdo (Caso número dos). 
La imagen descrita en la radiografía simple se trató de un DCG que dependía del borde mesentérico del colon, a nivel del tercio distal del colon transverso de aproximadamente $7.5 \mathrm{~cm} \times 6 \mathrm{~cm}$ de diámetro con un llenamiento parcial de medio de contraste (Figura 7). Debido a los hallazgos imagenológicos, se solicitó valoración por coloproctología, quienes consideraron que requería manejo quirúrgico y realizaron resección de colon transverso con anastomosis latero-lateral, con perforación contenida por epiplón.

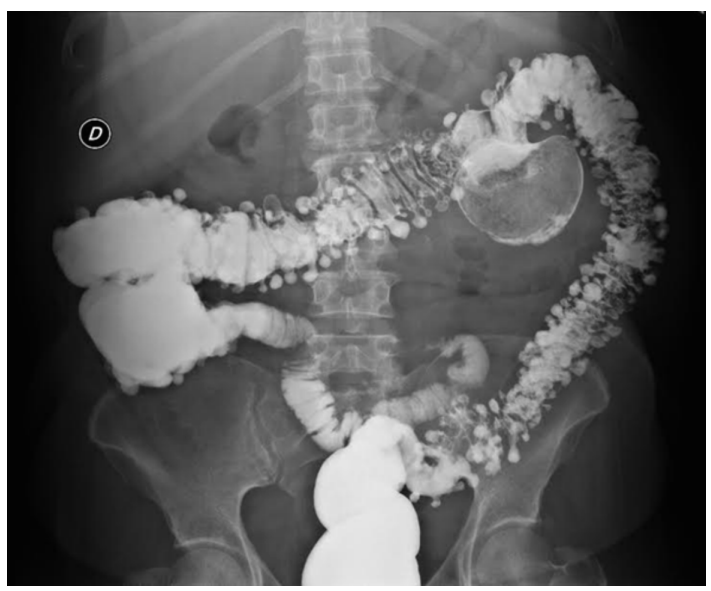

Figura 7. Colon por enema. Múltiples divertículos distribuidos en todo el colon. Divertículo colónico gigante localizado en el tercio distal del colon transverso de $7.5 \times 6 \mathrm{~cm}$ (Caso número dos)

\section{Discusión}

Los divertículos del colon son la protrusión sacular de la mucosa a través de áreas débiles de la pared muscular (1). Una presentación poco común es el divertículo colónico gigante, el cual se asocia a enfermedad diverticular en el $85 \%$ de los casos como se evidencia en el paciente número dos, aunque también pueden presentarse de manera aislada en un $15 \%$ como en el paciente número uno $(7,9)$. Se han descrito diversas clasificaciones, las más conocidas son las realizadas por McNutt et al (10) y Choong et al (11) (Tabla 1), basadas en la apariencia histológica $(5,8)$. En nuestros casos no fue posible obtener el reporte de patología por lo que no se pudieron clasificar.

La presentación clínica varía desde el paciente asintomático $(10 \%)$ en donde el DCG es descubierto de manera incidental como ocurrió en el paciente número 1, hasta el desarrollo de sintomatología crónica dada por distensión, dolor abdominal intermitente y estreñimiento; incluso puede presentarse como un abdomen agudo quirúrgico $(6,12)$. En el caso número dos la presentación fue aguda y esta se da en el $30-35 \%$ de los pacientes y se caracteriza principalmente por dolor abdominal, náuseas, emesis, fiebre, sensación de masa y deposiciones blandas (7).

Según la revisión realizada por Giuseppe Nigri et al (7), el dolor abdominal es el síntoma predominante en el $69 \%$ de los casos, seguido de constipación y sensación de masa abdominal con un $17 \%$ cada uno, emesis $12 \%$, y diarrea $11 \%$. De todos los pacientes incluidos en ese estudio el $26.5 \%$ presentó perforación como complicación, que fue diagnosticada en su mayoría en el momento de la cirugía, al igual que en el caso número dos. En cuanto a la exploración física el hallazgo más frecuentemente encontrado es la palpación de una masa abdominal como en el segundo caso $(12,13)$.

Debido a su infrecuente presentación, el diagnóstico depende principalmente de los hallazgos radiológicos como la radiografía simple, el colon por enema y la TAC que son las principales herramientas diagnósticas. Es importante conocer las características propias de cada método por cuanto permiten hacer un diagnóstico a tiempo y descartar oportunamente los diagnósticos diferenciales para poder llevar a cabo un buen tratamiento.

La ecografía por ser un método de fácil acceso se utiliza frecuentemente, sin embargo, no es el examen de elección para realizar un adecuado diagnóstico. No obstante, en el

Tabla 1. Clasificación del divertículo gigante de colon

\begin{tabular}{ccc}
\hline Tipo & Clasificación de McNutt (10) & Clasificación de Choong (11) \\
\hline I & Divertículo que aumenta de tamaño sin & Pseudo divertículo. Su pared es tejido fibroso \\
evidencia de perforación. En la pared se & e inflamatorio sin músculo liso con \\
pueden encontrar restos de muscularis \\
mucosae o muscularis propia y el \\
revestimiento interno tiene tejido de \\
granulación con restos de mucosa \\
colónica (22\%)
\end{tabular}

Fuente: Revista de Gastroenterología de México y modificada por los autores con sus valores respectivos de presentación (5). 
paciente número dos la ecografía fue de gran utilidad pues sugirió la presencia de una masa localizada en hipocondrio izquierdo.

La radiografía simple de abdomen es un método de elección costo-efectivo, en ella se observa una imagen radiolúcida redondeada de paredes finas, bien definidas adyacente al colon que puede contener un nivel hidroaérero en su interior $(7,14)$. Este método favorece la sospecha del DCG, sin embargo, no permite realizar un diagnóstico definitivo.

El enema de bario permite realizar el diagnóstico en la mayoría de los casos debido a que muestra la comunicación entre el DCG y el colon pues el contraste ingresa dentro del ostium, además, permite la visualización de diverticulosis asociada (Figura 7) (14).

La TAC es el examen diagnóstico de elección no solo debido a su alta sensibilidad, sino que también puede identificar la existencia de comunicación entre el divertículo y el tracto gastrointestinal $(4,6)$. Es un método no invasivo que permite visualizar el tamaño, la localización, el contenido y las paredes, además, es útil para mostrar la presencia de gas extraluminal si se ha producido perforación del divertículo $(6,14)$. Generalmente se observa una cavidad adyacente al colon de pared regular y fina, que puede contener líquido, aire o materia fecal sin realce del contraste (15) (Figura 1).

Para algunos autores el diagnóstico definitivo se realiza mediante el colon por enema, aunque su desventaja principal es que en ciertos pacientes no se evidencia la comunicación con el tracto gastrointestinal, por lo que la TAC sigue siendo el método de elección ante la sospecha de una masa abdominal sugestiva de DCG $(7,16)$ (Figura 8). La colonoscopia no contribuye al diagnóstico de esta patología debido a que el ostium del divertículo suele ser demasiado pequeño para permitir la visualización dentro de este y presenta un alto riesgo de perforación $(5,17)$.

Es muy importante tener en cuenta cuáles son las herramientas diagnósticas de elección que permiten una adecuada visualización del DCG ya que el retraso en el diagnóstico conlleva un tratamiento inadecuado por lo que se incrementa la tasa de morbimortalidad a causa de esta enfermedad (18).

Es necesario realizar un adecuado diagnóstico diferencial con los vólvulos del ciego donde se identifica una dilatación segmentaria de la pared de un asa con una colección de gas que se introduce en el hemiabdomen superior; el quiste de

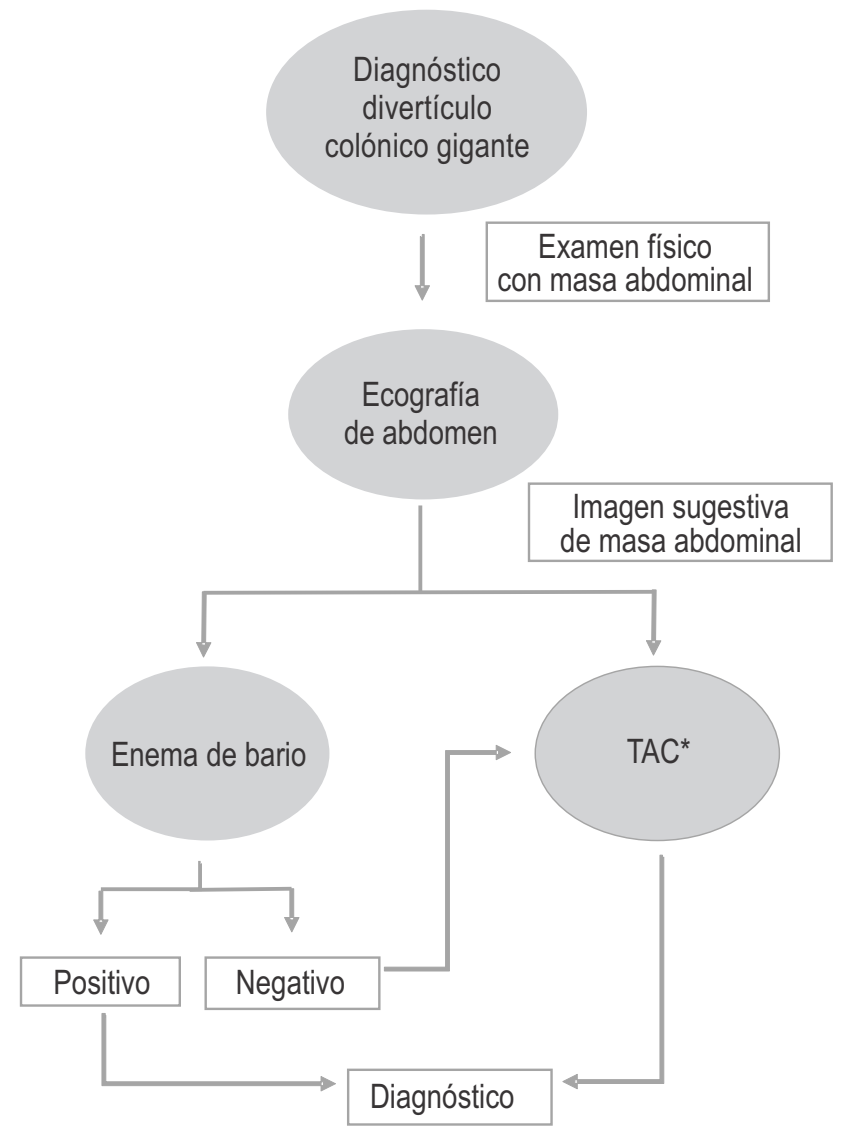

*TAC: Tomografía Axial Computarizada

Figura 8. Diagnóstico imagenológico del divertículo colónico gigante. 
duplicación entérica que se visualiza como una colección de gas que contiene las tres capas de la pared intestinal y se localiza en el borde mesentérico con predominio en pacientes jóvenes; el divertículo duodenal gigante que se observa como una hernia de la mucosa duodenal localizada en hemiabdomen derecho y ocupada por contraste en estudios digestivos; el divertículo de Meckel que se diagnostica tempranamente como una protrusión próxima al íleon terminal; los abscesos intrabadominales que se identifican como una lesión radiolúcida irregular y de aspecto moteado; los tumores necrosados o malignos como los sarcomas; el pseudoquiste pancreático y la colecistitis enfisematosa entre otras $(13,14)$.

La mayoría de estas entidades tienen una presentación clínica y hallazgos imagenológicos similares al DCG, sin embargo, existen características propias de cada una que permiten descartarlas o diagnosticarlas adecuadamente, en función de su localización, edad de presentación, sintomatología, exploración física e imágenes correspondientes como se realizó en los dos casos presentados. Según Giuseppe Nigri (8) del $15-35 \%$ de los pacientes se complican, siendo más frecuente la peritonitis, formación de abscesos, obstrucción intestinal, los vólvulos y el infarto intestinal $(5,6)$. La mayoría de las revisiones reportan que en el $2 \%$ de los casos se pueden desarrollar lesiones malignas dentro del divertículo $(8,19)$.

Respecto al tratamiento del DCG depende del contexto clínico y personal del paciente. Por ejemplo, los pacientes ancianos, asintomáticos y con un alto riesgo quirúrgico pueden ser manejados de manera conservadora con manejo antibiótico, drenaje percutáneo o implantación de stent en el cuello diverticular con drenaje del lumen $(3,8)$. Sin embargo, según Seenvoorde P (20) el tratamiento conservador puede traer complicaciones hasta en un $19 \%$ de los casos como obstrucción de asas delgadas, desarrollo de neoplasia maligna dentro del divertículo o una peritonitis por perforación en cualquier momento, por lo que recomienda esta terapia únicamente para pacientes con contraindicaciones absolutas para el manejo quirúrgico (20, $21,22)$.

El tratamiento de elección es la resección colónica y del divertículo con anastomosis colorectal primaria $(7,21)$. Según la revisión realizada por Giuseppe Nigri et al de los 166 casos expuestos en la literatura hasta este año al 57.2\% se le realizó este procedimiento, seguido por la resección colónica y del divertículo con colostomía tipo Hartman en el $11.4 \%$ la cual tiene como desventaja la necesidad de una intervención quirúrgica secundaria para restaurar la continuidad intestinal y finalmente la diverticulectomía que se realizó en el $10.2 \%$ de los pacientes, esta última representa un riesgo de dehiscencia por amplitud del cuello diverticular e inflamación del tejido circundante (8).

En los casos que informamos, el paciente número uno se manejó con tratamiento conservador con antibioticoterapia debido a que se encontraba asintomático en el momento de la valoración por cirugía general con adecuada evolución clínica por lo que se dio egreso. La paciente reportada en el caso número dos, por el contrario, presentó un cuadro clínico característico de esta entidad, se realizó el diagnóstico en el momento correcto y a pesar de sus patologías de base y de presentar perforación intraoperatoria contenida por epiplón, la cirugía se llevó a cabo satisfactoriamente.

En la Tabla 2 mencionamos las diferencias y similitudes más importantes para tener en cuenta comparando los dos

Tabla 2. Diferencias y similitudes entre los casos presentados y la literatura.

\begin{tabular}{|c|c|c|c|}
\hline & Caso no. 1 & Caso no. 2 & Literatura \\
\hline $\begin{array}{l}\text { Presentación } \\
\text { clínica }\end{array}$ & $\begin{array}{l}\text { Inició asintomático con } \\
\text { posterior aparición de } \\
\text { dolor abdominal, fiebre, } \\
\text { diarrea, astenia, } \\
\text { adinamia. }\end{array}$ & $\begin{array}{c}\text { Dolor abdominal, fiebre, diarrea, } \\
\text { náuseas, emesis. }\end{array}$ & $\begin{array}{l}\text { Asintomático (10\%) } \\
\text { Presentación aguda (30- } \\
\text { 35\%) caracterizada por } \\
\text { dolor abdominal, náuseas, } \\
\text { emesis, fiebre y diarrea. }\end{array}$ \\
\hline $\begin{array}{l}\text { Exploración } \\
\text { física }\end{array}$ & $\begin{array}{l}\text { Abdomen blando, no } \\
\text { masas, no irritación } \\
\text { peritoneal }\end{array}$ & $\begin{array}{l}\text { Masa } 5 \mathrm{~cm} \text { diámetro dolorosa, } \\
\text { signos de irritación peritoneal, } \\
\text { adenopatías inguinales } \\
\text { bilaterales. }\end{array}$ & $\begin{array}{l}\text { Palpación de masa } \\
\text { abdominal. }\end{array}$ \\
\hline Localización & Colon ascendente & Ángulo esplénico & Colon sigmoides $(90 \%)$ \\
\hline Diagnóstico & $\begin{array}{l}\text { TAC compatible con } \\
\text { DCG no asociado a } \\
\text { diverticulosis. }\end{array}$ & $\begin{array}{l}\text { Ecografía abdominal + TAC + } \\
\text { colon por enema compatible con } \\
\text { DCG asociado a diverticulosis }\end{array}$ & $\begin{array}{l}\text { Radiografía simple, colon } \\
\text { por enema y la TAC. }\end{array}$ \\
\hline Tratamiento & $\begin{array}{l}\text { Manejo conservador } \\
\text { con antibiótico-terapia } \\
\text { + nutrición parenteral }\end{array}$ & $\begin{array}{c}\text { Quirúrgico (Resección de colon } \\
\text { transverso con anastomosis } \\
\text { latero-lateral) }\end{array}$ & $\begin{array}{l}\text { La elección principal es la } \\
\text { cirugía, sin embargo, existe } \\
\text { manejo conservador. }\end{array}$ \\
\hline
\end{tabular}

* TAC: Tomografía Axial Computarizada, DCG: Divertículo colónico gigante. 
casos descritos y lo revisado en la literatura. En cuanto a la presentación clínica, la exploración física, la asociación con la enfermedad diverticular, el diagnóstico y el tratamiento los dos casos son concordantes con lo descrito anteriormente. Tuvimos un paciente asintomático y otro con presentación aguda, el hallazgo más importante, según el examen físico fue la palpación de una masa abdominal en uno de ellos, la asociación del DCG con enfermedad diverticular se dio en el paciente número dos, el diagnóstico se realizó por medio de imágenes tipo TAC y colon por enema y los dos tratamientos tanto el quirúrgico como el conservador fueron acordes con lo encontrado en la literatura. En cuanto a la localización los dos casos tuvieron ubicación diferente a lo encontrado comúnmente. Es importante resaltar que debido a la escases de presentación de esta entidad no se cuenta con una guía de abordaje, sin embargo, el paciente número uno se manejó de manera conservadora debido a sus antecedentes, su presentación clínica asintomática y la resolución adecuada del cuadro con antibioticoterapia y nutrición parenteral, por el contrario, el paciente numero dos tuvo una clínica aguda con signos de irritación peritoneal lo cual requirió intervención por parte de coloproctología quienes consideraron oportuno el tratamiento quirúrgico, lo que está acorde con el manejo de elección descrito en estudios anteriores.

\section{Conclusiones}

El DCG es una entidad poco frecuente y por tanto poco descrita en la literatura asociada en su mayoría con la diverticulosis. Se presenta con una clínica muy diversa sugestiva de múltiples patologías abdominales que deben ser descartadas para poder realizar un adecuado diagnóstico por medio de técnicas de imagen como la radiografía, el colon por enema y la Tomografía Axial Computarizada (TAC) que representan las principales herramientas diagnósticas de esta entidad.

El tratamiento de elección es la intervención quirúrgica, sin embargo, en contextos determinados como pacientes ancianos, asintomáticos y con alto riesgo quirúrgico puede manejarse de manera conservadora. Su importancia a pesar de la baja prevalencia radica en la necesidad del diagnóstico oportuno para evitar una alta tasa de complicaciones como la perforación, la obstrucción de asas, los vólvulos y el infarto intestinal. Los dos casos presentados fueron abordados de manera diferente, y cada uno tuvo una presentación clínica y hallazgos imagenológicos diferentes; sin embargo, se manejaron de acuerdo con lo descrito en la literatura.

\section{Responsabilidades éticas}

Protección de personas y animales. Los autores declaran que para esta investigación no se han realizado experimentos en seres humanos ni en animales.
Confidencialidad de los datos. Los autores declaran que han seguido los protocolos de su centro de trabajo sobre la publicación de datos de pacientes.

Derecho a la privacidad y consentimiento informado. Los autores han obtenido el consentimiento informado de los pacientes referidos en el artículo. Este documento obra en poder del autor de correspondencia.

\section{Conflicto de intereses}

Los autores declaran no tener conflicto de interés.

\section{Referencias}

1. Medina F, Díaz N, Gallardo A, Gómez I, Garcilazo D, Gómez J. Nuevas tendencias en el manejo de la diverticulitis y la enfermedad diverticular del colon. Rev Esp Enferm Dig. 2015; 107(3):162-170.

2. Murphy T, Hunt R, Fried M, Krabshuis J. Enfermedad Diverticular [Internet]. World Gastroenterology Organization Practice Guidelines. 2004. Disponible en: http://www.worldgastroenterology.org/UserFiles/file/gui delines/diverticular-disease-spanish-2004.pdf

3. Mahamid A, Ashkenazi I, Sakran N, Zeina A. Giant colon diverticulum: rare manifestation of a common disease. Isr Med Assoc J. 2012; 14(5):331-332.

4. Ochoa E, Esquivel C, Badra R, Cornet M, Marangoni A. Divertículo gigante de colon. Presentación de un caso. Rev Argent Radiol. 2008; 72(4):423-428.

5. Weber L, Bravo C, Garteiz M, Carbó R, Vega F. Divertículo colónico gigante. Informe de un caso y revisión de la bibliografía. Rev Gastroenterol Mex. 2010; 2(75):213-217.

6. Zeina A, Mahamid A, Nachtigal A, Ashkenazi I, Shapira M. Giant colonic diverticulum: radiographic and MDCT characteristics. Insights into imaging. 2015; 6(6):659-64

7. Nigri, G, Petrucciani N, Giannini G, Aurello P, Magistri P, Gasparrini M, et al. Giant colonic diverticulum: clinical presentation, diagnosis and treatment: systematic review of 166 cases. World J Gastroenterol. 2015; 21(1):360-368.

8. Olakowski M, Jabłońska B, Lekstan A, Szczęsny W, Pilch J, Kohut M. Gastrointestinal Image: A True Giant Transverse Colon Diverticulum. J Gastrointest Surg. 2011; 15:1289-1291.

9. Singh A, Raman S, Brooks C, Philips D, Desai R, Kandarpa K. Giant colonic diverticulum: percutaneous computed tomography-guided treatment. J Comput Assist Tomogr. 2008; 32(2):204-206.

10. McNutt R, Schmitt D, Schulte W. Giant colonic diverticula-three distinct entities. Report of a case. Dis Colon Rectum. 1988; 31(8):624-628.

11. Choong C, Frizelle F. Giant colonic diverticulum: report of four cases and review of the literature. Dis Colon Rectum. 1998; 41(9):1178-1185.

12. Gorodner, A, Juárez H, Borda G, Disanto $O$, Gorodner A. Divertículo gigante de Colon Sigmoides: presentación de dos casos y consideraciones sobre el manejo de esta 
rara complicación de la enfermedad diverticular. Universidad Nacional del Nordeste. 2006; 25(1):26-27.

13. Iglesias G, Sánchez R, Peña J. Solution to case 36. Giant diverticulum of the sigmoid colon. Elsevier/ Radiología. 2011; 53(6):575-577.

14. Navarro E, Carro B, Castillo C, Lasierra R. Divertículo gigante de colon. Radiología. 2006; 48(5):313-5.

15. Thomas S, Peel R, Evans L, Haarer K. Best cases from the AFIP: Giant colonic diverticulum. Radiographics. 2006; 26(6):1869-1872.

16. Fuentes E, Díaz J, Serrano E, Maldonado M. Divertículo gigante de colon. Presentación de un caso. Rev Cubana Cir. 2007; 46(1).

17. Silva C, Cortés C. Caso Radiológico. Rev hil radiol. 2006; 12(2):104-105.
18. González J, Menéndez J. Divertículo sigmoideo gigante complicado en un paciente diabético. Rev Cubana Cir 2013; 52(3)205-210.

19. Mohammad A, Ben-Nakhi A, Khoursheed M. Giant sigmoid diverticulum: a case report. Med Princ Pract. 2009; 18(1):70-72.

20. Steenvoorde P, Vogelaar F, Oskam J, Tollenaar R. Giant colonic diverticula. Review of diagnostic and therapeutic options. Dig Surg. 2004; 21(1):1-6.

21. Kim H, Kim J, Moon O, Kim K. Giant ascending colonic diverticulum presenting with intussusception. Ann Coloproctol. 2013; 29(5):209-212.

22. Durgakeri P, Strauss P. Giant sigmoid diverticulum: $A$ case report. Australas Med J. 2015; 8(3):85-88. 